\title{
Healthy conversation skills: increasing competence and confidence in front-line staff
}

\author{
Christina Black ${ }^{1,2 *}$, Wendy Lawrence ${ }^{1}$, Sue Cradock ${ }^{2}$, Georgia Ntani ${ }^{1}$, Tannaze \\ Tinati $^{1}$, Megan Jarman ${ }^{1,2}$, Rufia Begum ${ }^{1,2}$, Hazel Inskip ${ }^{1}$, Cyrus Cooper ${ }^{1}$, Mary Barker ${ }^{1}$ \\ and Janis Baird ${ }^{1}$ \\ 'Medical Research Council Lifecourse Epidemiology Unit (University of Southampton), Southampton General \\ Hospital, Tremona Road, Southampton SO 16 6YD, UK: ${ }^{2}$ NIHR Biomedical Research Unit in Nutrition, Diet and \\ Lifestyle, Southampton University NHS Hospitals Trust, Southampton, UK
}

Submitted 16 January 2012: Final revision received 11 June 2012: Accepted 1 August 2012: First published online 19 September 2012

\begin{abstract}
Objective: (i) To assess change in confidence in having conversations that support parents with healthy eating and physical activity post-training. (ii) To assess change in staff competence in using 'open discovery' questions (those generally beginning with 'how' and 'what' that help individuals reflect and identify barriers and solutions) post-training. (iii) To examine the relationship between confidence and competence post-training.

Design: A pre-post evaluation of 'Healthy Conversation Skills', a staff training intervention.

Setting: Sure Start Children's Centres in Southampton, England.

Subjects: A total of 145 staff working in Sure Start Children's Centres completed the training, including play workers (43\%) and community development or family support workers (35\%).

Results: We observed an increase in median confidence rating for having conversations about healthy eating and physical activity (both $P<0 \cdot 001$ ), and in using 'open discovery' questions $(P<0 \cdot 001)$, after staff attended the 'Healthy Conversation Skills' training. We also found a positive relationship between the use of 'open discovery' questions and confidence in having conversations about healthy eating post-training $(r=0 \cdot 21, P=0 \cdot 01)$, but a non-significant trend was observed for having conversations about physical activity $(r=0 \cdot 15, P=0 \cdot 06)$.

Conclusions: The 'Healthy Conversation Skills' training proved effective at increasing the confidence of staff working at Sure Start Children's Centres to have more productive conversations with parents about healthy eating. Wider implementation of these skills may be a useful public health nutrition capacity building strategy to help community workers support families with young children to eat more healthy foods.
\end{abstract}

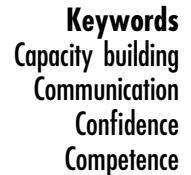

A mother's diet quality influences fetal and infant diet and health, and has subsequent implications for risk of chronic disease later in life ${ }^{(1-3)}$. The recent public health white paper 'Healthy Lives, Healthy People: Our Strategy for Public Health in England' places importance on optimising maternal health, particularly for mothers from disadvantaged backgrounds ${ }^{(4)}$. The white paper also highlights the need for preventive initiatives in early years services to enhance the health and well-being of mothers and their children. Sure Start Children's Centres in the UK are a Government initiative that aims to provide health, education and support services for young children and their families, where emphasis is placed on reaching the most vulnerable ${ }^{(5)}$. Healthy eating is identified as a target issue for Sure Start Children's Centres; many centre activities and services provide an opportunity to expose children and parents to healthy foods and support healthy dietary behaviours ${ }^{(6)}$. Ensuring staff working in these centres are equipped with core skills in public health nutrition, particularly in supporting behaviour change, has the potential to improve the diets and long-term health of women and children.

Workforce development is a key strategy in building public health nutrition capacity in individuals, organisations and communities to effectively address population nutrition issues ${ }^{(7)}$. The importance of up-skilling front-line health and community workers to enhance the reach, effectiveness and sustainability of community-based interventions is widely recognised in the fields of health promotion, public health and public health nutrition ${ }^{(8,9)}$. 
In a recent assessment of expert consensus on public health nutrition competency units for Europe, $98 \%$ of respondents agreed or strongly agreed that workforce development for public health nutrition should be multidisciplinary and include front-line staff such as community nurses $^{(10)}$. The vast majority of expert respondents also agreed that basic-intermediate level competence in behaviour change and health promotion methods is necessary for front-line staff.

A key aim of workforce development initiatives is to increase staff confidence or self-efficacy in a particular skill or set of skills. Self-efficacy is defined as belief in one's ability or competence to undertake a particular action $^{(11)}$ and it is common for confidence measures to be used in training evaluations to measure trainees' change in self-efficacy ${ }^{(12-14)}$. Practice self-efficacy has been described as a cognitive measure of the effects of a training initiative, indicating staff intent or perceived competence to adopt or implement a new skill ${ }^{(15)}$. These authors proposed that it is important to enhance staff practice self-efficacy because it is positively associated with the implementation of new skills. Thus increasing staff confidence can be an important workforce development initiative.

It has been argued that competencies are the architecture for workforce development because they codify the skills, knowledge and attitudes required for effective performance in the workplace ${ }^{(9)}$. Previous research in Southampton Sure Start Children's Centres found staff had strong relationships and engaged well with families ${ }^{(16)}$. However, opportunities for discussion and problem solving about healthy eating were frequently missed. Observations of staff practice demonstrated that staff members were not supporting parents to change their dietary and physical activity habits. It was decided by the local management team that practice could be enhanced if staff groups were offered training to increase their confidence and competence in having conversations to motivate and sustain positive behaviour change ${ }^{(16)}$.

Consequently, a behaviour change training intervention, 'Healthy Conversation Skills', was developed and delivered to staff working in Sure Start Children's Centres in Southampton. This training was designed to enable staff to have more productive conversations with parents attending their centres by encouraging them to reflect on their dietary and physical activity habits and find their own solutions to their issues. The training was based on a philosophy of empowerment and encompassed an adult-learning, problem-solving approach. The training consisted of three group sessions focusing on five competencies. A summary of the training and the five key competencies the training was designed to increase is provided in Box 1, and a full description of the training intervention has been published elsewhere ${ }^{(17)}$.

The current paper describes the effectiveness of the training intervention in increasing staff confidence in having conversations with parents about healthy eating and

\section{Box 1 'Healthy Conversations Skills' training}

Communication is enhanced through training staff in the use of asking open-ended, or 'open discovery', questions: those that generally begin with 'how' and 'what'. Such conversations allow an individual to explore an issue, identify barriers and generate solutions that can be reviewed with staff at their next encounter. 'Healthy Conversation Skills' training aims to increase self-efficacy and sense of control of both staff and the people they work with.

The five competences addressed are:

A. identifying and creating opportunities to hold 'healthy conversations';

B. using open-ended ('open discovery') questions; C. reflecting on practice;

D. listening more than talking; and

E. supporting individually derived goal setting through SMARTER planning (Specific, Measurable, Action-oriented, Realistic, Timed, Evaluated, Reviewed).

'Healthy Conversations Skills' training consists of three $3 \mathrm{~h}$ group sessions over 3 to 5 weeks to allow time for practising new skills and reflecting on practice. Training is delivered by a team of researchers experienced in group work and behaviour change. Training is followed by a period of ongoing support, including a telephone call and one $3 \mathrm{~h}$ follow-up workshop approximately 4 to 12 weeks and more than 3 months after training, respectively. These activities allow trainees to reflect on the training, how they have implemented their new skills and the barriers that got in the way of implementation.

physical activity, and in increasing staff competence in using 'open discovery' questions. The paper also explores the relationship between competence and confidence.

\section{Methods}

'Healthy Conversation Skills' training was delivered by a training team consisting of public health nutritionists and psychologists to staff working in the fourteen Sure Start Children's Centres in Southampton, a relatively deprived city in the affluent south of England. Southampton is ranked as the eighty-first most deprived local authority in England out of 354 such areas ${ }^{(18)}$. The majority of activities aimed at improving the diets and physical activity levels of young women living in disadvantaged areas of Southampton are delivered through Sure Start Children's Centres.

\section{Participants}

Between May 2009 and January 2011, all play, family support and community development workers employed 
by Sure Start Children's Centres in Southampton were required to attend 'Healthy Conversation Skills'. Administrative staff, community health nurses and oral health workers were also invited to voluntarily attend the training (Table 1). All managers and coordinators of these centres were invited to voluntarily attend a one-day 'taster' session of 'Healthy Conversation Skills'; a total of thirteen attended who consisted of $81 \%$ of all management ( $n$ 16). All training activities were delivered during staff working hours.

The local ethics committee did not require a submission for this work. The training programme and associated evaluation were undertaken as part of staff continuing professional development. All trainees completed a registration sheet and consented to take part in the training.

\section{Evaluation process and materials}

Data were collected at three time points (Fig. 1). All staff who participated in the 'Healthy Conversation Skills' training completed a time one (T1) and time two (T2) evaluation sheet at the beginning of session 1 and end of session 3, respectively. To measure change in competence in using 'open discovery' questions, trainees recorded responses to four written statements made by parents about difficulties with healthy eating and physical activity. Participants provided individual written responses to these statements mimicking what their verbal response would have been if a parent had made this comment to them while they were working. Specifically, these statements were: 'There are lovely vegetables outside the shops, but I don't know what they are'; 'I can't afford for us to join a gym'; 'It's more never

Table 1 Job roles of staff who attended the 'Healthy Conversation Skills' training

\begin{tabular}{lcr}
\hline Job role & No. of staff trained & $\%$ \\
\hline Play supervisor/play worker & 63 & 43 \\
Community development worker & 26 & 18 \\
Family support worker & 24 & 17 \\
Community health nurse & 16 & 11 \\
Administrative staff/project worker & 9 & 6 \\
Oral health practitioner & 6 & 4 \\
Manager/coordinator & 1 & 1 \\
Total & $145^{\star}$ & 100 \\
\hline
\end{tabular}

${ }^{*}$ Three of these trainees were men and 142 were women. being taught what to eat, to cook or whatever'; and 'I just don't seem to have time to do any exercise'. The training team developed a coding matrix to code and score objectively trainee responses to the four statements. The coding matrix consisted of seven possible coding options. Responses were scored according to the level to which they would allow a parent to reflect on their difficulties and identify their own solution. Giving information by telling or making suggestions was given the lowest score of 1 , while asking 'open discovery' questions to help reflect and plan for change was given the highest score of 6 (Table 2). A total score for all four responses was calculated for each participant. The number of times 'open discovery' responses were used by each trainee (minimum of 0 and maximum of 4) was also used as a measure of competence in the analysis. Responses were double-coded by two trainers to ensure consistency of coding. Coding discrepancies were reviewed by the two trainers and discussed until an agreement was reached.

Change in perceived confidence and importance of staff in having conversations with parents about healthy eating and physical activity while at work was also measured between $\mathrm{T} 1$ and $\mathrm{T} 2$. The measure of importance was included to gauge the priority staff members give to healthy eating and physical activity as two of many issues they discuss with parents and to determine their level of receptivity to training in skills that support behaviour change around these topics. The measures of confidence and importance consisted of three 10-point Likert scale questions, where 1 was the lowest score of confidence and importance, and 10 was the highest. Specifically, the three questions read: 'How confident do you feel about having conversations with individuals about eating healthily?'; 'How confident do you feel about having conversations with individuals about being physically active?'; and 'How important is it for you to help individuals change their diets and physical activity levels?'. An overall confidence score was derived for each individual by calculating the mean of their healthy eating and physical activity confidence scores. The relationship between level of confidence and competence in using 'open discovery' questions was analysed using overall confidence scores, which were divided into three levels: low (score $=1-3$ ), mid (score $=4-7$ ) and high (score $=8-10)$ confidence.

All trainees received a follow-up telephone call between 4 and 12 weeks after their third training session. The length

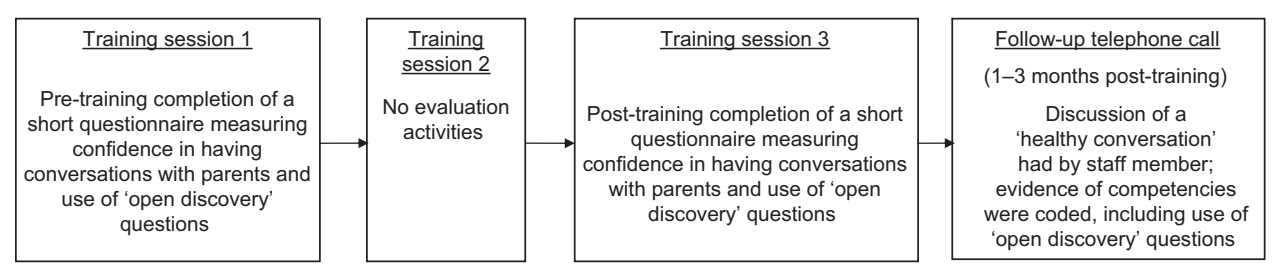

Fig. 1 Data collection process 
Table 2 Abridged coding matrix to assess and score trainees' use of 'open discovery' questions

\begin{tabular}{ll}
\hline Response score & Example \\
\hline 0. Unable to code & $\begin{array}{l}\text { Find out if they know about courses } \\
\text { and give them a leaflet } \\
\text { Missing data (no response) }\end{array}$ \\
$\begin{array}{l}\text { 1. Telling/suggestion } \\
\text { (giving information) }\end{array}$ & $\begin{array}{l}\text { How about walking to work? } \\
\text { I found that too, this is what I usually } \\
\text { do... }\end{array}$ \\
2. In my experience & $\begin{array}{l}\text { That must be difficult } \\
\text { 3eems like you'd really like to eat more } \\
\text { vegetables } \\
\text { Do you feel you have to go to a gym to } \\
\text { exercise more? }\end{array}$ \\
4. Closed question & $\begin{array}{l}\text { Why can't you fit that into your life? } \\
\text { What have you tried? How could you } \\
\text { change this? }\end{array}$ \\
\hline $\begin{array}{l}\text { 5. Other open question } \\
\text { 6. 'Open discovery' } \\
\text { question }\end{array}$ & \\
\hline
\end{tabular}

of time between completion of training and follow-up telephone calls varied as some staff were difficult to contact because of the part-time and sessional nature of their roles, and because of extended breaks during school holidays. The follow-up telephone calls provided an opportunity for trainees to reflect on their application of 'Healthy Conversation Skills', offer feedback about their experience of the training and enabled trainers to assess trainee competence. Trainers followed a semi-structured script to indentify use of 'open discovery' questions in practice. The script began with trainers asking: 'Can you tell me about a conversation you've had where you used the skills we talked about in the "Healthy Conversation Skills" training? How did the conversation start? What was the first thing you said?'. The trainer continued to use prompts such as 'What else did you say?' and 'Who did most of the talking in the conversation?' to elicit details about the trainees' level of competence in using 'open discovery' questions to help parents explore and plan for change to help improve their lifestyle behaviours. All telephone calls were audio-recorded, transcribed and conversations double-coded by trainers using a coding matrix developed to assess demonstrated level of competence in each of the five competencies (Box 1). Trainees were awarded a level of competence from 0 to 4 , where 0 represented no demonstration of competency and 4 represented strong demonstration of competency. The present paper focuses on competency B - use of 'open discovery' questions.

\section{Statistical analysis}

Importance of and confidence in having conversations about healthy eating and physical activity, and competence scores, were described using medians and interquartile ranges (IQR). Changes in importance of and confidence in having healthy eating and physical activity conversations pretraining and post-training were tested using the Wilcoxon matched-pairs signed-rank test. Spearman correlation was used to assess pre- and post-training associations between

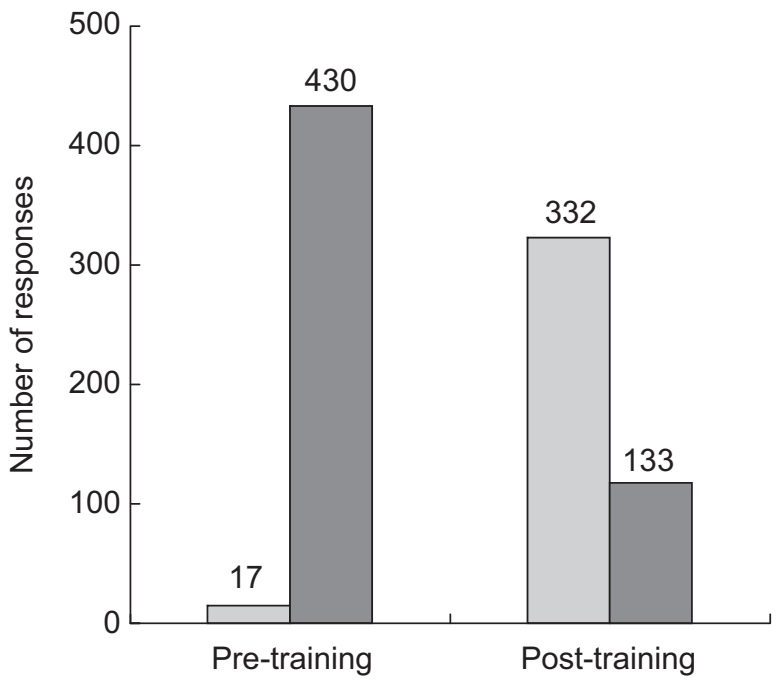

Fig. 2 Staff use of 'open discovery' questions ( $\square$ ) and telling/ suggestions $(\square)$ before and after attending the 'Healthy Conversation Skills' staff training intervention; staff ( $n$ 145) working in Sure Start Children's Centres, Southampton, UK. There was a significant change in the use of both 'open discovery' questions and telling/suggestions between pre- and post-training (both $P<0.001$ )

'open discovery' questions total score and confidence score. Data were analysed using the STATA statistical software package version $11 \cdot 1$.

\section{Results}

\section{Staff characteristics}

A total of 209 staff working across fourteen Sure Start Children's Centres in Southampton were eligible to take part in the 'Healthy Conversation Skills' training. Of these, 148 attended the training, giving a reach of $71 \%$, and 145 provided complete evaluation data for analyses reported in the present paper. Of all trainees, 142 (98\%) were women. Table 1 shows that the largest staff group who attended were play workers or play supervisors ( $43 \%$ ), followed by community development workers (18\%) and family support workers (17\%). Fewer health workers (11\% nursing and $4 \%$ oral health) and administrative staff (6\%) participated in the training. One centre manager also attended the 'Healthy Conversation Skills' training.

\section{Staff competence: immediately post-training}

Staff use of 'open discovery' questions changed as a result of attending the 'Healthy Conversation Skills' training. Prior to the training, the vast majority of responses to the four statements were in the form of providing information (telling) or making suggestions. However, after the training the majority of responses were in the form of 'open discovery' questions (Fig. 2). These changes in responses pre- and post-training were statistically significant $(P<0 \cdot 001)$. Of those trainees ( $n$ 131) who asked no 'open discovery' 
Table 3 The relationship between overall confidence* and competence in using 'open discovery' questions in response to four statements post-training; 'Healthy Conversation Skills' training intervention among staff $(n 145)$ working in Sure Start Children's Centres, Southampton, UK

\begin{tabular}{|c|c|c|c|c|c|}
\hline \multirow[b]{2}{*}{ Overall confidence } & \multicolumn{5}{|c|}{ Competence in using 'open discovery' questions } \\
\hline & None & $1-2$ times & 3-4 times & Total & $P$ \\
\hline Low & 1 & 0 & 0 & 1 & $0.002 \uparrow$ \\
\hline Mid & 15 & 21 & 30 & 66 & \\
\hline High & 11 & 18 & 49 & 78 & \\
\hline Total & 27 & 39 & 79 & 145 & \\
\hline
\end{tabular}

Results presented are numbers of participants.

*Overall confidence scores were derived for each participant by calculating the mean of his/her healthy eating and physical activity confidence scores.

tSpearman test for trend across increasing competence and overall confidence levels $(r=0 \cdot 19)$.

questions pre-training, $27 \%$ were using one or two 'open discovery' questions and $53 \%$ were using three or four after the training. Only $20 \%$ of participants did not use any 'open discovery' questions after the training. The barriers staff face to implementing 'open discovery' questions are described elsewhere ${ }^{(19)}$.

\section{Staff competence: follow-up}

We conducted follow-up telephone calls with all trainees between 4 and 12 weeks after they completed the training to assess their level of competence in using 'open discovery' questions in practice. Complete data were available for 136 trained staff members. During the telephone call the staff member recounted a 'healthy conversation' he/she had had since attending the training. Level of competency was graded from 0 , no demonstration of competency, to 4 , strong demonstration of competency. Data showed that $49 \%$ of staff were demonstrating high competence (score $=3$ or 4 ) in using 'open discovery' questions more than a month after they had completed the 'Healthy Conversations Skills' training. In contrast, no competence $($ score $=0)$ was demonstrated by $6 \%$ of staff.

\section{Staff confidence}

Prior to attending the 'Healthy Conversation Skills' training, Sure Start Children's Centre staff reported moderate levels of confidence in having conversations with parents about healthy eating and physical activity (both: median 7, IQR 5-8). At the end of the training the median level of staff confidence increased to 8 (IQR 7-9) and 8 (IQR 7-8) for healthy eating and physical activity respectively; in both these variables change in staff confidence was found to be statistically significant (both $P<0 \cdot 001$ ). Posttraining $57 \%$ of staff increased in confidence, $22 \%$ decreased and $21 \%$ showed no change in having conversations about healthy eating; $52 \%$ of staff increased confidence, $28 \%$ decreased and $20 \%$ showed no change in conversations about physical activity.

\section{Staff competence and competence immediately post-training}

We examined the relationship between staff competence and confidence both pre- and post-training. Pre-training, we found no relationship between competence in 'open discovery' question total score and confidence in having conversations about healthy eating $(r=0 \cdot 13, P=0 \cdot 13)$ or physical activity $(r=0 \cdot 09, P=0 \cdot 28)$. Post-training, we found a significant positive correlation between competence in 'open discovery' question total score and level of confidence in having conversations about healthy eating $(r=0 \cdot 21, P=0 \cdot 013)$; however, the association was not quite significant for physical activity $(r=0 \cdot 15, P=0 \cdot 06)$.

Table 3 illustrates the relationship between low, mid and high overall confidence levels and competence in using 'open discovery' questions post-training. We found a positive relationship between level of competence and overall confidence $(r=0 \cdot 19, P=0 \cdot 02)$. The majority of staff reported high overall confidence $(54 \%)$ in having conversations with parents about healthy eating and physical activity and most of those who reported high overall confidence demonstrated high competence in using 'open discovery' questions (63\%) post-training. Only one member of staff reported having low overall confidence, and this one individual displayed no competence in using 'open discovery' questions post-training. The group of staff who reported mid-level overall confidence in having conversations with parents about healthy eating and physical activity displayed varying levels of competence in using 'open discovery' questions, where $23 \%$ showed no competence ( score $=1-3), 32 \%$ mid-level competence $($ score $=4-7)$ and $45 \%$ high competence (score $=8-10$ ).

\section{Importance of bealthy eating and physical activity}

Sure Start Children's Centre staff reported that helping parents change their diets and physical activity levels was of high importance (median 8, IQR 6-10). There was no change in median levels of reported importance following the training (median 8, IQR 7-9).

\section{Discussion}

The vast majority of Sure Start Children's Centre staff demonstrated increased competence in using 'open discovery' questions immediately after attending 'Healthy 
Conversation Skills'. Our data also showed that staff considered healthy eating and physical activity to be important issues to discuss with parents of young children and that their confidence in having conversations about healthy eating and physical activity with parents increased after attending the training. These findings suggest that 'Healthy Conversations Skills' training was successful at increasing the capacity of front-line staff in Children's Centres to support parents to make positive behaviour changes by asking questions to help them identify their own solutions to their lifestyle issues rather than giving suggestions or telling parents what they should do. Analysis of the follow-up telephone call data showed that almost half of the trained staff continued to demonstrate high competency in using 'open discovery' questions in practice over a month after they attended the training. This finding indicates that staff were implementing the skill in the workplace and suggests the 'Healthy Conversations Skills' training produces sustained changes in staff practice. We anticipated that a significant positive association would develop between competence and confidence after the training but observed this relationship only for having conversations about healthy eating. The relationship for confidence in having conversations about physical activity fell just outside established significance levels.

The present study is the first one to assess the association between competence in a key public health nutrition skill and level of confidence in using that particular skill. Assessing the relationship between confidence and competence is important because it goes beyond the assumption that a staff training intervention has been effective if either staff confidence or competence has increased; a practitioner may have increased his/her confidence but may not have demonstrated competence in the key skills. Equally, a practitioner may have demonstrated competence but not increased his/her confidence in implementing the skill. The significant positive relationship between competence and overall confidence we observed after the training may be a more powerful indication that a training intervention will lead to positive changes in staff practice. Our measure of competence in using 'open discovery' questions at follow-up assessed sustained competence and sustained use of the skill within the workplace. The evaluation tools used to measure competence, however, were developed by the training team and have not been validated for use in other settings. Furthermore, this training focused on staff working at Sure Start Children's Centres and therefore results may not be generalisable outside this group. None the less, trainees represented a wide variety of staff roles and all had regular contact with the community. It is a limitation that the current study did not collect background information about trainees such as age, educational attainment, years of work experience in the field or previous behaviour change/communications training. The 'Healthy Conversations Skills' training was delivered as part of routine continuing professional development and such data are not usually collected in these situations. The finding in the current study that training in a key public health nutrition and health promotion skill increases the confidence of staff to use that skill is consistent with previous public health and health promotion workforce development studies ${ }^{(20,21)}$. While our measure of competence was based on staff self-report rather than more objective measures, it was measured on more than one occasion, including more than a month after the training. We have used level of confidence as a proxy for staff practice self-efficacy because it is a widely used approach for measuring change in practice selfefficacy of staff in workforce development research ${ }^{(12-14)}$.

The shift in staff competence in using 'open discovery' questions rather than telling or making suggestions that we observed in the present study is similar to a training intervention in South Africa which aimed to improve the health promotion skills of front-line health staff in motivating individuals and empowering them to make informed health choices $^{(22)}$. The results of that qualitative study showed that many of the trainees described moving from being prescriptive advisors or information disseminators to facilitating behaviour change by asking questions and listening more when interacting with clients. The data thus infer that practitioners increased their competence in behaviour change communication skills.

The increase in staff confidence or practice self-efficacy that we observed in the present study is similar to previous workforce development research which found a significant increase in staff practice self-efficacy following training of front-line health professionals in health promotion principles and strategies ${ }^{(21,22)}$. Findings from a mentoring intervention based on the principles of Appreciative Inquiry also showed increased confidence in key public health nutrition skills after the intervention ${ }^{(20)}$. The authors concluded that having the opportunity to critically reflect on practice was a successful strategy for increasing staff confidence to reorient their practice. The 'Healthy Conversation Skills' training was based on a philosophy of empowerment and problem solving, where the design of the training provided trainees with time to critically reflect on their practice and try using the new skills in the workplace. It is likely that these opportunities for reflection and practice enabled the development of a positive feedback loop for staff, whereby those who used the new skills grew more in confidence ${ }^{(15)}$. While reflective practice is seen as an effective workforce development technique ${ }^{(23)}$, it may not always have a positive impact on confidence. In our study a proportion of staff showed a decrease in confidence in having conversations with parents about healthy eating and physical activity after the training. This finding suggests that some staff may lag in the development and implementation of learning new skills and highlights the importance of coupling staff training activities with organisational support that encourages staff to practise and build their confidence in applying the new skills ${ }^{(24)}$. 
As far as we are aware, our study is the only public health nutrition capacity building study to assess the association between competence in a key practice skill and level of confidence in using that particular skill. Our study data identified a positive association between competence in using 'open discovery' questions and staff confidence in having conversations about healthy eating after the training. Similar findings were observed for conversations about physical activity; however, the results failed to reach significance. We can provide no objective explanation for this situation because the training activities focused on supporting parents to improve both healthy eating and physical activity behaviours. The slight discrepancy in these findings may be artificial; however, if genuine, they may suggest that the relationship between confidence and competence involves a complex interaction and may depend on the subject areas being explored. It may be possible that staff perceived physical activity to be a relatively simple behaviour change message and that their role in supporting parents to be more active is clear. By contrast, there may have been confusion around healthy eating behaviour change messages, where even simple messages such as '5-a-day' may cause confusion; for example, knowing how much a portion of fruit or vegetables is and how the portion sizes differ for adults and children. It is possible that staff felt they were lacking in knowledge about healthy eating prior to training, but after attending 'Healthy Conversation Skills' felt less pressure in having to tell or suggest to parents accurate information and felt more able to support parents in finding achievable solutions to their healthy eating issues. The disparity of these findings is being further explored qualitatively through focus groups with trained staff.

Staff reported that it was important for them to help parents to improve their diet and physical activity behaviours. Staff working in Sure Start Children's Centres are therefore likely to be receptive to professional development opportunities in public health nutrition, a conclusion further supported by the high number of employees who attended the 'Healthy Conversation Skills' training. Although healthy eating and physical activity are highlighted as target issues for Sure Start Children's Centres, little is known about effective nutrition training or development opportunities for staff in this context. Considering the population health benefits of improving the diets and lifestyles of mothers and their children, ensuring professional development opportunities for children's centre staff are effective is an essential component of increasing public health nutrition capacity ${ }^{(25)}$.

Workforce development strategies alone, however, are unlikely to make significant contributions to workforce capacity unless combined with supporting strategies including leadership, adequate resourcing and organisational change ${ }^{(7)}$. It has been argued that building skills among individual practitioners will not lead to effective practice without strong support from the organisation within which they work ${ }^{(22)}$. Key components of organisational change include involving all levels of staff in the change process in order to overcome resistance and involving both senior and middle managers as key catalysts for change by acting as role models for staff ${ }^{(24)}$. Institutional constraints have been noted as a barrier to implementing new health promotion skills, particularly when managers' understanding of the training conflicts with staff members' understanding, largely as a result of managers not attending the training ${ }^{(22)}$. In our study the vast majority of managers and coordinators were involved in one-day pilot sessions of 'Healthy Conversation Skills'. This exposure to the training was informative for managers and enabled them to support staff development in the new skills.

While there has been a push in the fields of public health and health promotion for outcome-focused workforce development initiatives that are based on agreed competency frameworks and adult-learning principles ${ }^{(26,27)}$, guidance is lacking about how best to measure level of practitioner competence. These competency frameworks are useful for directing the content of workforce development initiatives; however, tools to measure practitioner change in competences such as behaviour change communication skills have typically been based on general statements $^{(10,20,28)}$ that do not break down the competency into specific behaviours that can be measured. The methods developed for our study may be helpful for future researchers and practitioners wanting to measure the specific behaviours that demonstrate competence in having conversations that support behaviour change.

\section{Conclusion}

'Healthy Conversation Skills' training proved effective at increasing the confidence of staff working at Children's Centres to have more productive conversations with parents about healthy eating and at improving their competence in using 'open discovery' questions. While the relationship between competence and confidence is not clearly understood, it appears that increasing staff competence in communication skills that support healthy behaviour changes is associated with staff being more confident to engage in conversations with parents about healthy eating. Implementation of 'Healthy Conversation Skills' may be a useful public health nutrition capacity building strategy to help community workers support families with young children to eat more healthy foods. The impact this training intervention has on the quality of women and children's diets is currently being evaluated.

\section{Acknowledgements}

Sources of funding: The study was funded by the Medical Research Council (UK), NIHR Biomedical Research Unit (Nutrition, Diet and Lifestyle) Southampton University 
NHS Hospitals Trust and NHS Southampton City. Conflicts of interest: There are no conflicting interests. Author contributions: J.B., M.B., C.C. and H.I. contributed to the study design. C.B., W.L. and S.C. delivered the training and in collaboration with T.T., M.J. and R.B. evaluated the training data. G.N. assisted with the statistical analysis. C.B. drafted the manuscript and all authors made contributions. Acknowledgements: The authors extend their thanks to the Sure Start Children's Centre staff and managers in Southampton who participated in the 'Health Conversation Skills' training and evaluation activities, to Southampton City Council for its engagement and to Greg Deakin for his work with data entry and cleaning.

\section{References}

1. Bruce KD \& Hanson MA (2010) The developmental origins, mechanisms, and implications of metabolic syndrome. J Nutr 140, 648-652.

2. Robinson S, Marriott L, Poole J et al. (2007) Dietary patterns in infancy: the importance of maternal and family influences on feeding practice. Br J Nutr 98, 1029-1037.

3. Barker DJ (1997) Fetal nutrition and cardiovascular disease in later life. Br Med Bull 53, 96-108.

4. Department of Health (2010) Healthy Lives, Healthy People: Our Strategy for Public Health in England. London: Her Majesty's Stationery Office.

5. Belsky J, Barnes J \& Melhuish E (2007) The National Evaluation of Sure Start: Does Area-Based Early Intervention Work? Bristol: Policy Press.

6. Latham P, Kapoor S, Myers P et al. (2006) Breastfeeding, Weaning and Healthy Eating: A Synthesis of Sure Start Local Programme Evaluation Findings. Birkbeck: Institute for the Study of Children, Families \& Social Issues.

7. Baillie E, Bjarnholt C, Gruber M et al. (2009) A capacitybuilding conceptual framework for public health nutrition practice. Public Health Nutr 12, 1031-1038.

8. Hawe P, Noort M, King L et al. (1997) Multiplying health gains: the critical role of capacity-building within health promotion programs. Health Policy 39, 29-42.

9. Hughes R (2008) Workforce development: challenges for practice, professionalization and progress. Public Health Nutr 11, 765-767.

10. Jonsdottir S, Hughes R, Thorsdottir I et al. (2011) Consensus on the competencies required for public health nutrition workforce development in Europe - the JobNut project. Public Health Nutr 14, 1439-1449.

11. Bandura A (1997) Self Efficacy: The Exercise of Control. New York: Freeman.

12. Hallford DJ, McCabe MP, Mellor D et al. (2011) Intervention for depression among palliative care patients and their families: a study protocol for evaluation of a training program for professional care staff. BMC Palliat Care 10, 11.
13. Ammentorp J \& Kofoed PE (2011) Research in communication skills training translated into practice in a large organization: a proactive use of the RE-AIM framework. Patient Educ Couns 82, 482-487.

14. Lenzi R, Baile WF, Costantini A et al. (2011) Communication training in oncology: results of intensive communication workshops for Italian oncologists. Eur J Cancer Care 20, 196-203.

15. Turner KMT, Nicholson JM \& Sanders MR (2011) The role of practitioner self-efficacy, training, program and workplace factors on the implementation of an evidence-based parenting intervention in primary care. J Prim Prev 32, 95-112.

16. Lawrence W, Keyte J, Tinati $\mathrm{T}$ et al. (2012) A mixedmethods investigation to explore how women living in disadvantaged areas might be supported to improve their diets. J Health Psychol 17, 785-798.

17. Barker M, Baird J, Lawrence W et al. (2011) The Southampton Initiative for Health: a complex intervention to improve the diets and increase the physical activity levels of women from disadvantaged communities. J Health Psychol 16, 178-191.

18. Southampton City Council (2011) Index of Multiple Deprivation 2010. http://www.southampton.gov.uk/living/ statsresearch/imd2010.aspx (accessed September 2011).

19. Tinati T, Lawrence W, Ntani G et al. (2012) Implementation of new Healthy Conversation Skills to support lifestyle changes - what helps and what hinders? Experiences of Sure Start Children's Centre staff. Health Soc Care Community 20, 430-437.

20. Palermo C, Hughes R \& McCall L (2010) An evaluation of a public health nutrition workforce development intervention for the nutrition and dietetics workforce. J Hum Nutr Diet 23, 244-253.

21. Devine SG, Llewellyn-Jones L \& Lloyd J (2009) Impact of a five-day short course on integration of health promotion into practice in north Queensland. Health Promot J Aust 20, 69-71.

22. Wills J \& Rudolph M (2010) Health promotion capacity building in South Africa. Glob Health Promot 17, 29-34.

23. Wenger E (2000) Communities of practice and social learning systems. Organization 7, 225-246.

24. Yeatman HR \& Nove T (2002) Reorienting health services with capacity building: a case study of the Core Skills in Health Promotion Project. Health Promot Int 17, 341-350.

25. Hughes R (2006) A socioecological analysis of the determinants of national public health nutrition work force capacity: Australia as a case study. Fam Community Health 29, 55-67.

26. Koo D \& Miner K (2010) Outcome-based workforce development and education in public health. Annu Rev Public Health 31, 253-269.

27. Van der Zanden GH, Barry MM, Battel-Kirk B et al. (2010) Developing pan European competency-based professional standards and accreditation framework for health promotion. Eur J Public Health 20, 235.

28. Hughes R (2004) Competencies for effective public health nutrition practice: a developing consensus. Public Health Nutr 7, 683-691. 